\title{
Knockdown of hsa_circ_0091994 constrains gastric cancer progression by suppressing the miR-324-5p/HMGA1 axis
}

\author{
Yi Xie ${ }^{1}$, Zhao Liu ${ }^{1}$, Hanfang Zhu ${ }^{1}$ \\ ${ }^{1}$ Department of Gastrointestinal Surgery, Henan Provincial People's Hospital, Zhengzhou 450000, Henan, China
}

Correspondence to: Yi Xie; email: xieyi032@126.com, https://orcid.org/0000-0002-1311-3245

Keywords: hsa_circ_0091994 (cicrRNA_105040), gastric cancer, miR-324-5p, apoptosis, migration

Received: January 4, $2021 \quad$ Accepted: May 14, $2021 \quad$ Published: August 24, 2021

Copyright: (c) 2021 Xie et al. This is an open access article distributed under the terms of the Creative Commons Attribution License (CC BY 3.0), which permits unrestricted use, distribution, and reproduction in any medium, provided the original author and source are credited.

\begin{abstract}
CircRNAs have emerged as potential therapeutic targets for diseases such as gastric cancer (GC). We identified highly dysregulated circRNAs in GC tissue and further explored their potential mechanisms in the progression of GC. Hsa_circ_0091994 (cicrRNA_105040) was identified as a highly upregulated circRNA in GC tissues, whose host gene is negatively associated with the overall survival of patients. Using cell counting kit-8 and Annexin V assays, we observed that hsa_circ_0091994 knockdown inhibited the viability of AGS and HGC-27 cells by inducing apoptosis. Scratch wound healing assays showed that hsa_circ_0091994 knockdown also inhibited GC cell healing. Bioinformatics analysis and a luciferase assays revealed that hsa_circ_0091994 knockdown inhibits GC progression by suppressing miR-324-5p and HMGA1 expression. The antitumor effect of hsa_circ_0091994 knockdown was confirmed in vivo using a mouse xenograft model. Hsa_circ_0091994 knockdown inhibited the progression of GC by inhibiting the miR-324-5p/HMGA1 axis.
\end{abstract}

\section{INTRODUCTION}

Gastric cancer (GC) is a prevalent malignancy with nearly 1 million new cases per year globally [1-4]. China has the highest incidence of GC, and more than $50 \%$ of cases occur in East Asia [5-7]. GC is the third leading cause of cancer mortality worldwide with over 720,000 deaths annually [7, 8]. Surgery remains the primary treatment strategy [3], but the 5-year survival rate is only $20-50 \%$ with surgery alone [9]. Even with multimodal treatment, the 5-year overall survival rate for patients with advanced GC is less than 5\% [3].

Dysregulation of several genes and pathways are correlated to the pathogenesis of GC $[10,11]$. Circular RNA (circRNA) is a class of endogenous RNA with covalently-closed loop structures [12]. Back-spliced exons generate circRNAs throughout the eukaryotic transcriptome [13]. During the post-transcriptional process, circRNAs can disrupt miRNAs [14]. CircRNAs stimulate GC cell proliferation, apoptosis, migration, invasion, and epithelial mesenchymal transition (EMT)
$[15,16]$. CircRNAs also promote the initiation, development, differentiation, metastasis, and TNM stage of GC tumors $[15,17]$.

Using the Gene Expression Omnibus (GEO) dataset, we compared the expression profiling of circRNAs between GC tissues and normal tissues. Differentially expressed circRNAs (DEcircRNAs) of GSE141977 were explored in vitro and in vivo.

\section{RESULTS}

CircRNA hsa_circ_0091994 is increased in GC tissue

We identified dysregulated circRNAs in GC tissues using the GSE141977 dataset (Figure 1A, 1B). The association between the host genes of these dysregulated circRNAs and the survival rate of patients with GC was analyzed. FLNA, the host gene of hsa_circ_0091994 (circ_105040), was negatively correlated to the survival rate of patients with GC (Figure 1C). 
Hsa_circ_0091994 knockdown repressed cell viability of AGS and HGC-27

Levels of hsa_circ_0091994 in GES-1, GAS, MKN45, and HGC-27 cells were detected by RT-qPCR. Hsa_circ_0091994 expression was increased in three GC cancer cell lines compared with GES-1 (Figure 2A). Compared with MKN45, hsa_circ 0091994 had higher levels in AGS cells and HGC-27 cells. Therefore, AGS and HGC-27 cells were selected for exploring the role of hsa_circ_0091994 in vitro.
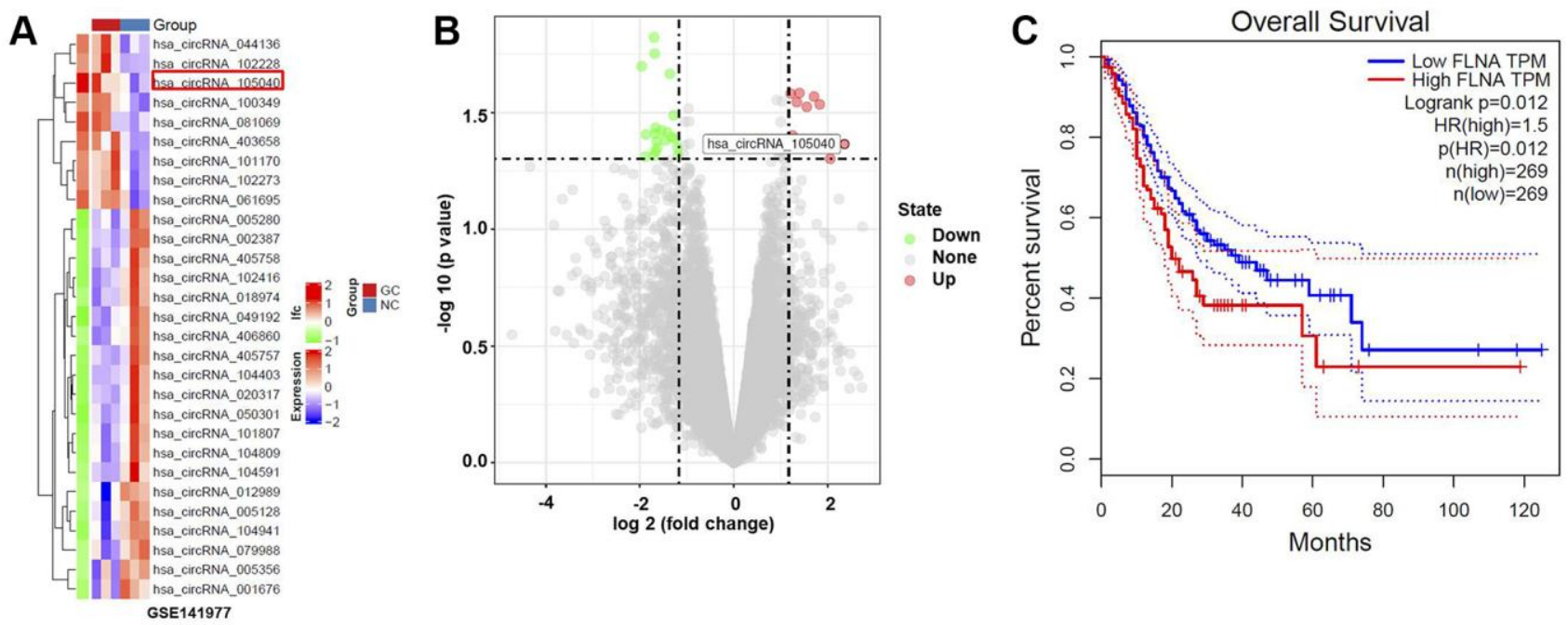

Figure 1. Bioinformatics analysis to identify DEcircRNAs in GC. (A) Heat map indicating DEcircRNAs in GC tissue. (B) Volcano plot of DEcircRNAs. Highly downregulated and upregulated genes indicated in green and red, respectively. P-value $<0.05\left(-\log _{10} \mathrm{p}\right.$-value $\left.>1.3\right)$ and $\mid \log _{2}$ (fold change) $\mid>1.5$ were thresholds. (C) Survival analysis demonstrating the relationship between the level of FLNA (the host gene of hsa_circ_0091994) and survival rate of GC patients.
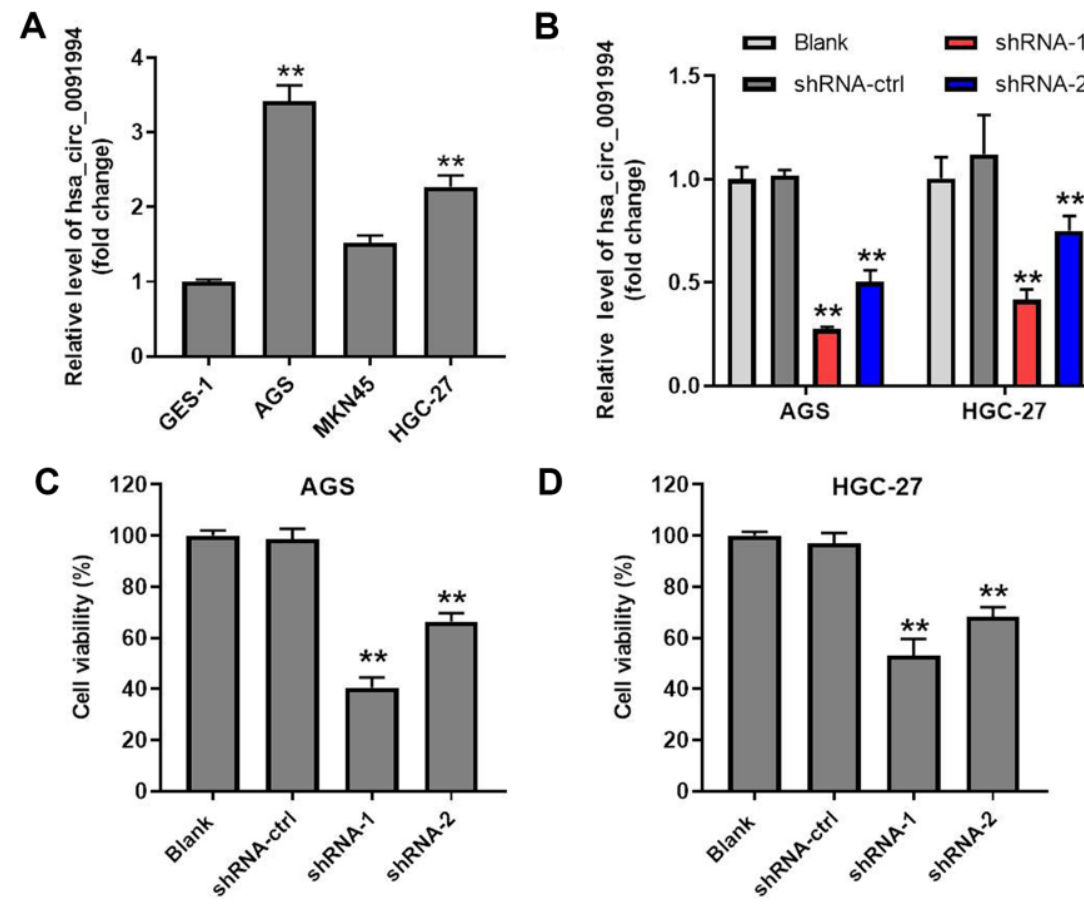

Figure 2. Hsa_circ_0091994 knockdown inhibited cell viability of AGS and HGC-27. (A) The relative level of hsa_circ_0091994 in human gastric epithelial cell line GES-1 and in GC cell lines including AGS, MKN45, and HGC-27. ${ }^{*} * \mathrm{P}<0.01$, compared with GES-1; $\mathrm{n}=3$. (B) AGS and HGC-27 cells were administrated with shRNA-ctrl, shRNA-1 or shRNA-2 of hsa_circ_0091994 for 48 hr. The efficacy of hsa_circ_0091994 knockdown was evaluated by RT-qPCR. (C, D) The effect of shRNA-1 or shRNA-2 of hsa_circ_0091994 on the viability of AGS and HGC-27 cells were determined by CCK- 8 assay. ${ }^{*} * P<0.01$, compared with blank; $n=3$. 
Two shRNAs (shRNA1 and shRNA2) were designed and synthesized to knockdown hsa_circ_0091994 in AGS cells and HGC-27 cells, and knockdown efficacy was confirmed with RT-qPCR (Figure 2B). Cell viability assay was performed at $48 \mathrm{hr}$ after transfection, which showed that both hsa_circ_0091994 shRNAs suppressed gastric cancer cell viability (Figure 2C, 2D and Supplementary Figure 1A). However, hsa_circ_0091994 shRNA-1 exhibited a stronger inhibition of cell viability and was carried forward into the next experiments. In contrast, overexpression of hsa_circ_0091994 significantly promoted the proliferation of gastric cancer cells (Supplementary Figure 1B, 1C).

\section{Hsa_circ_0091994 knockdown induced apoptosis and inhibited wound healing activity in AGS and HGC-27 cells}

We used Annexin V and PI double staining to assess the effect of hsa_circ_0091994 knockdown on GC cell apoptosis. Compared with shRNA-ctrl group, shRNA-1 stimulated apoptosis in gastric cancer cells (Figure 3A, 3B and Supplementary Figure 1D). In addition, the wound healing activity of AGS and HGC-27 cells were inhibited by hsa_circ_0091994 shRNA-1 (Figure 3C, 3D). Meanwhile, the expression of EMT associated makers in AGS cells was detected with western blot. The result indicated hsa_circ_0091994 knockdown increased the level of E-cadherin and decreased the level of $\alpha$-SMA (Figure 3E-3G). Taken together, hsa_circ_0091994 knockdown induced apoptosis and inhibited wound healing activity in AGS and HGC-27 cells.

\section{Hsa_circ_0091994 functions through regulating miR-324-5p/HMGA1 axis}

In order to investigate the underlying mechanisms by which hsa_circ_0091994 regulated GC progression, the putative targets of hsa_circ_0091994 were predicted using StarBase and CircInteractome databases.
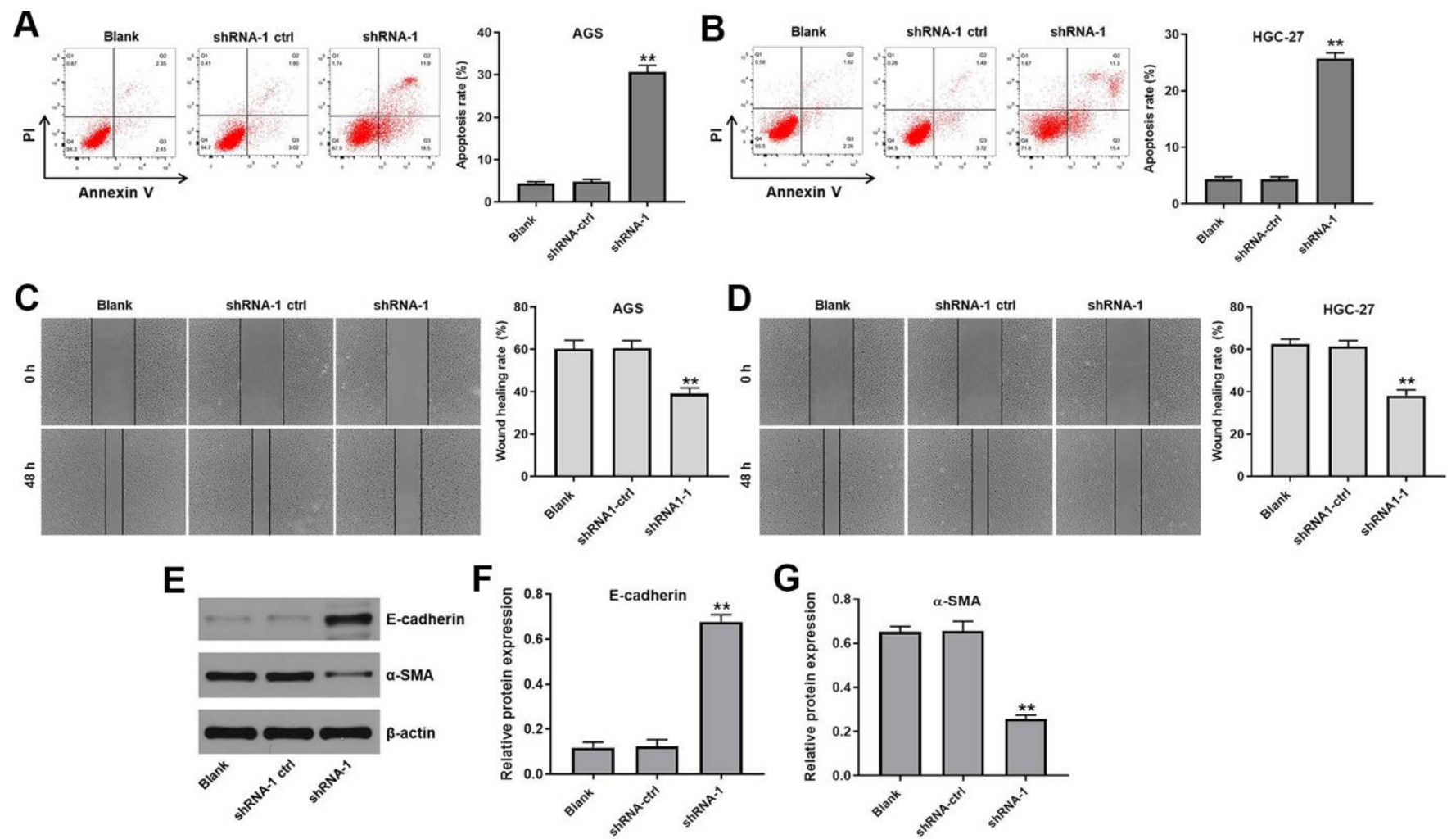

Figure 3. Hsa_circ_0091994 knockdown promoted the apoptosis and suppressed the wound healing activity in AGS and HGC-27 cells. AGS and HGC-27 cells were treated with shRNA-1 or shRNA-ctrl for 48 hr. (A, B) Cell apoptosis rate was measured in each group. (C, D) Wound healing assay was employed to evaluate the effect of hsa_circ_0091994 knockdown on wound healing activity of AGS and HGC-27 cell. (E) The protein expression of E-cadherin and $\alpha$-SMA was examined using western blot assay. $\beta$-actin was used as a loading control. (F, G) The expressions of E-cadherin and $\alpha$-SMA were quantified respectively. The**P<0.01, compared with blank; $\mathrm{n}=3$. 
MiR-324-5p and miR-217 were predicted as potential targets of hsa_circ_0091994 (Figure 4A). Among these two miRNAs, miR-324-5p is correlated with GC progression [18], so we investigated the relationship between hsa_circ_0091994 and miR-324-5p. Wild type and mutant 3' UTR of hsa_circ_0091994 were listed and synthesized (Figure 4B), and miR-324-5p agomir or negative control (agomir control) was transfected into the cells (Figure 4C). The relative luciferase activity of wild type hsa_circ_0091994 was decreased by miR324-5p agomir, indicating that miR-324-5p was the target of hsa_circ_0091994 (Figure 4D). In addition, the result of RNA pull-down indicated miR-324-5p directly binding to hsa_circ_0091994 (Supplementary Figure 2A). Moreover, miR-324-5p antagomir reversed the effects of hsa_circ_0091994 knockdown on cell viability and apoptosis (Supplementary Figure 2B-2D).

We further explored the downstream target of miR-3245p. HMGA1 was predicted as one of the putative downstream targets of miR-324-5p (Figure 4E), and HMGA1 promotes GC progression [19]. Relative luciferase activity of wild type HMGA1 was decreased by miR-324-5p agomir (Figure 4F), suggesting HMGA1 is a downstream target of miR-324-5p.

HMGA1 expression in GC tissue and adjacent normal tissue was analyzed using GEPIA tool based on the expression profile from TCGA database [20]. Bioinformatics analysis showed that HMGA1 was highly expressed in GC tumor tissue (Figure 4G). In addition, the expressions of hsa_circ_0091994 and HMGA1 in clinical gastric tumor tissues were notably upregulated, while the level of miR-324-5p was downregulated compared with that in adjacent tissues (Figure 4H-4J). All these data suggested hsa_circ_0091994 functions through regulating miR324-5p/HMGA1 axis.

\section{HMGA1 OE reversed the effects of hsa_circ_0091994 knockdown on cell viability and apoptosis}

Rescue experiments were performed to verify the function of hsa_circ_0091994 in GC cells. Since hsa_circ_0091994 knockdown inhibited GC cell
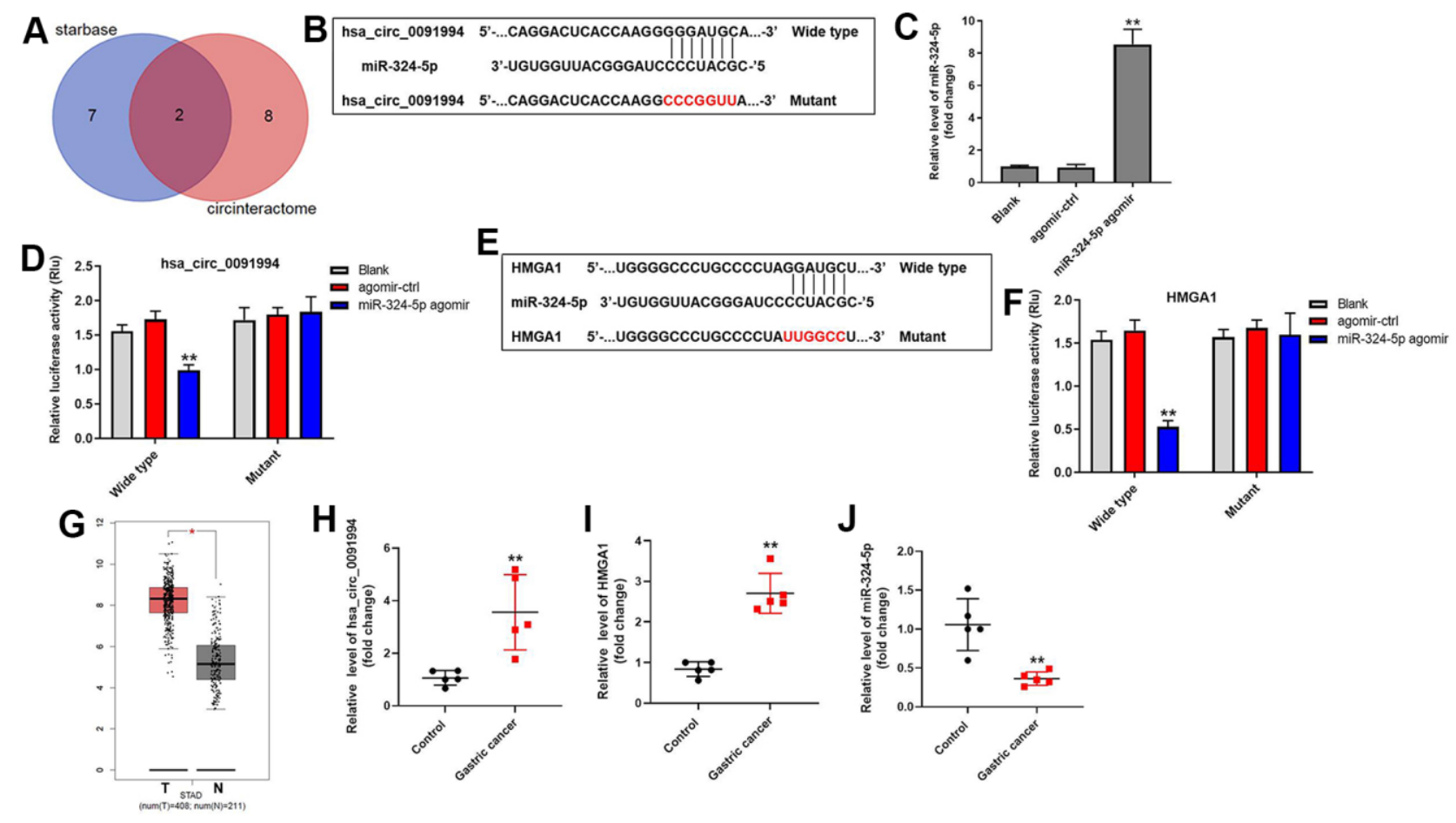

Figure 4. Downstream target of hsa_circ_0091994. (A) The downstream targets of hsa_circ_0091994 were predicted using Starbase and Circlnteractome. (B) MiR-324-5p was predicted as the downstream target of hsa_circ_0091994. Sequences of wild type and mutant 3' UTR of miR-324-5p. (C) MiR-324-5p agomir or the negative control (agomir-ctrl) was transfected into AGS cells for $48 \mathrm{hr}$. The efficacy of miR324-5p agomir was detected by RT-qPCR. (D) Dual luciferase assay for hsa_circ_0091994 was conducted at $48 \mathrm{hr}$ after transfection. (E) HMGA1 was predicted as the downstream target of miR-324-5p. Sequences of wild type and mutant 3' UTR of HMGA1 were presented. (F) Dual luciferase assay for HMGA1 was conducted at $48 \mathrm{hr}$ after transfection. (G) Bioinformatics analysis using GEPIA tool was performed to analyze the levels of HMGA1 in GC tissue and normal gastric tissue. (H-J). The expressions of hsa_circ_0091994, miR-324-5p and HMGA1 in 5 pairs of clinical GC and adjacent normal tissues were detected with RT-qPCR. ${ }^{* *} \mathrm{P}<0.01$, compared with blank; $\mathrm{n}=3$. 
proliferation via suppressing the miR-324-5p/HMGA1 axis, HMGA1 overexpression (OE) and shRNA-1 vectors were co-transfected into AGS cells. HMGA1 OE reversed the decrease in cell viability that was induced by shRNA-1 (Figure 5A). Similarly, HMGA1 $\mathrm{OE}$ reversed the increase in apoptosis that was induced by shRNA-1 (Figure 5B). Protein expression of HMGA1, Bax, Bcl-2, and cleaved caspase-3 was examined by western blot (Figure 5C). The shRNA-1 decreased HMGA1 and Bcl-2 expression, and increased Bax and cleaved caspase 3 levels (Figure 5D-5G). However, HMGA1 OE reversed all of these effects (Figure 5C-5G).

\section{Hsa_circ_0091994 knockdown inhibited tumor growth in mouse xenograft model}

Finally, the role of hsa_circ_0091994 was explored using with a mouse xenograft model. Tumor growth was suppressed by hsa_circ_0091994 knockdown at 5 weeks (Figure 6A). Tumor tissue was harvested and weighed right after sacrifice. Tumors in the shRNA-1 group were smaller than those in the control group (Figure 6B). In addition, the levels of hsa_circ_0091994 and HMGA1 in the shRNA-1 group were notably downregulated, while miR-324-5p level was slightly upregulated (Figure 6C). These data indicated hsa_circ_0091994 knockdown inhibited GC tumor growth in vivo.

\section{DISCUSSION}

Gastric cancer (GC) patients typically have a poor prognosis [3, 4, 7, 9], and some circRNAs are biomarkers and therapeutic targets for cancer treatments [15-17]. We investigated hsa_circ_0091994 in vitro and in vivo because its parental gene FLNA expression was negatively associated with the overall survival of GC patients.

We found miR-324-5p was the downstream miRNA of hsa_circ_0091994 in GC, and increased miR-324-5p can inhibit GC progression [18]. Using StarBase database, we found HMGA1 to be the direct target of miR-324-5p, and HMGA1 has oncogenic activity in multiple cancers, including GC [21, 22]. Cell viability assay and a xenograft model showed that HMGA1 overexpression could reverse the anti-tumor effect of hsa_circ_0091994 knockdown in GC.

Hsa_circ_0091994 knockdown inhibited GC progression by suppressing the miR-324-5p/HMGA1 axis, which suggests hsa_circ_0091994 might serve as a novel therapeutic target for the treatment of GC.
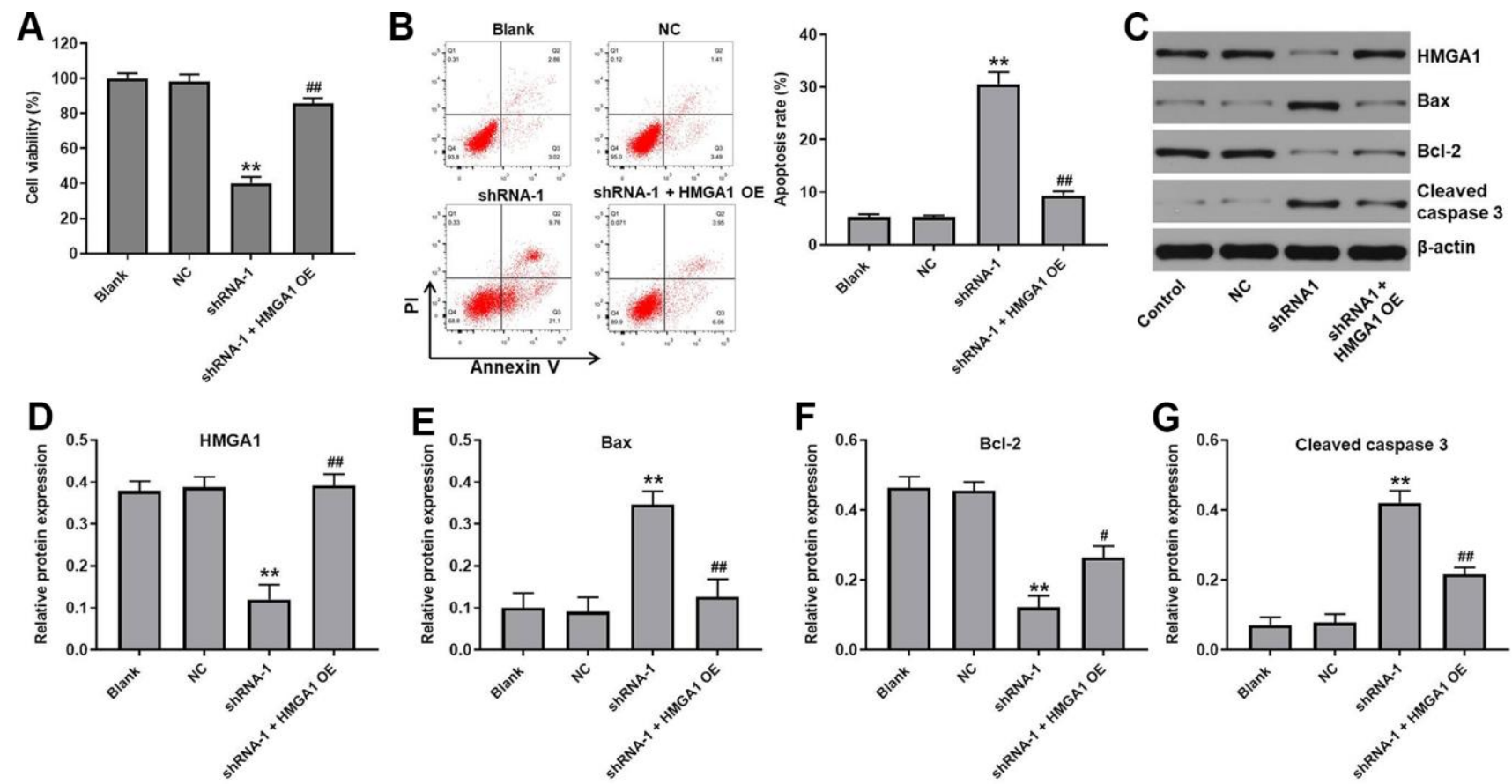

Figure 5. HMGA1 OE reversed the effect of hsa_circ_0091994 knockdown on the cell viability and apoptosis. AGS cells were administrated with shRNA-1 and HMGA1 OE for 48 h. (A) Cell viability was detected using CCK-8 assay. (B) Cell apoptosis was detected using Annexin V and PI double staining; cell apoptosis rate was quantified. (C) The protein expression of HMGA1, Bax, Bcl-2, cleaved caspase 3 was examined using western blot assay. $\beta$-actin was used as a loading control. (D-G) The protein expressions of HMGA1, Bax, Bcl-2, and cleaved caspase 3 were quantified respectively. ${ }^{*} \mathrm{P}<0.01$, compared with blank; $\mathrm{n}=3$. 


\section{MATERIALS AND METHODS}

\section{Bioinformatics analysis}

The circRNAs expression data of GC tissues and matched normal tissues data were downloaded from the Gene Expression Omnibus (GEO, https://www.ncbi. nlm.nih.gov/geo/) under the accession number GSE141977. The differentially expressed circRNAs (DEcircRNAs) between GC tissues and normal tissues were analyzed and identified using $\mathrm{R}$. P-value $<0.05$ $\left(-\log _{10}\right.$ p-value $\left.>1.3\right)$ and $\mid \log _{2}$ Fold Change $\mid>1.5$ was set as the threshold of significant DEcircRNAs. FLNA is the parental gene of hsa_circ_0091994. The association between the level of FLNA and the survival rate of patients with GC was analyzed using TCGA dataset (http://tcga-data.nci.nih.gov/tcga). GIEPIA (http://gepia.cancer-pku.cn/about.html) tool was employed to analyze the level of HMGA1 in GC tissue and normal gastric tissue.

\section{Cell culture}

Human gastric epithelial cell line GES-1 was obtained from Beyotime (Shanghai, China). Gastric cancer cell line AGS was purchased from American Type Culture Collection (ATCC, Manassas, VA, USA). Gastric cancer cell line MKN45 and HGC-27 cells were obtained from Shanghai Institutes for Biological Sciences (SIBS, Shanghai, China). All cells were maintained in RPMI1640 medium (Thermo Fisher Scientific, Waltham, MA, USA) supplemented with $10 \% \mathrm{FBS}, 100 \mathrm{U} / \mathrm{mL}$ penicillin, and $100 \mathrm{mg} / \mathrm{mL}$ streptomycin in a humidified $5 \% \mathrm{CO}_{2}$ incubator at $37^{\circ} \mathrm{C}$.

\section{Sample collection}

The gastric cancer tissues and adjacent normal tissues were isolated from 5 patients with gastric cancer in Henan Provincial People's Hospital. Tissues were isolated and stored in liquid nitrogen immediately. The study was approved by the Ethics Committee of Henan Provincial People's Hospital, and the written informed consent from patient was documented.

\section{Reverse transcription-quantitative PCR (RT-qPCR)}

Total RNA Extraction Reagent (ELK Biotechnology, Wuhan, China) was used to extract total RNA from the cells and tissues. The related data of the tumor tissues were presented in Figure 6A-6C. Then, EntiLink ${ }^{\mathrm{TM}}$ 1st Strand cDNA Synthesis Kit (ELK Biotechnology) was employed for reverse transcription. The qPCR reactions were carried

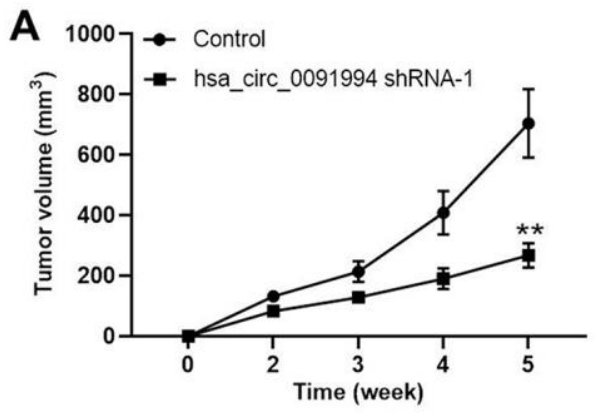

B
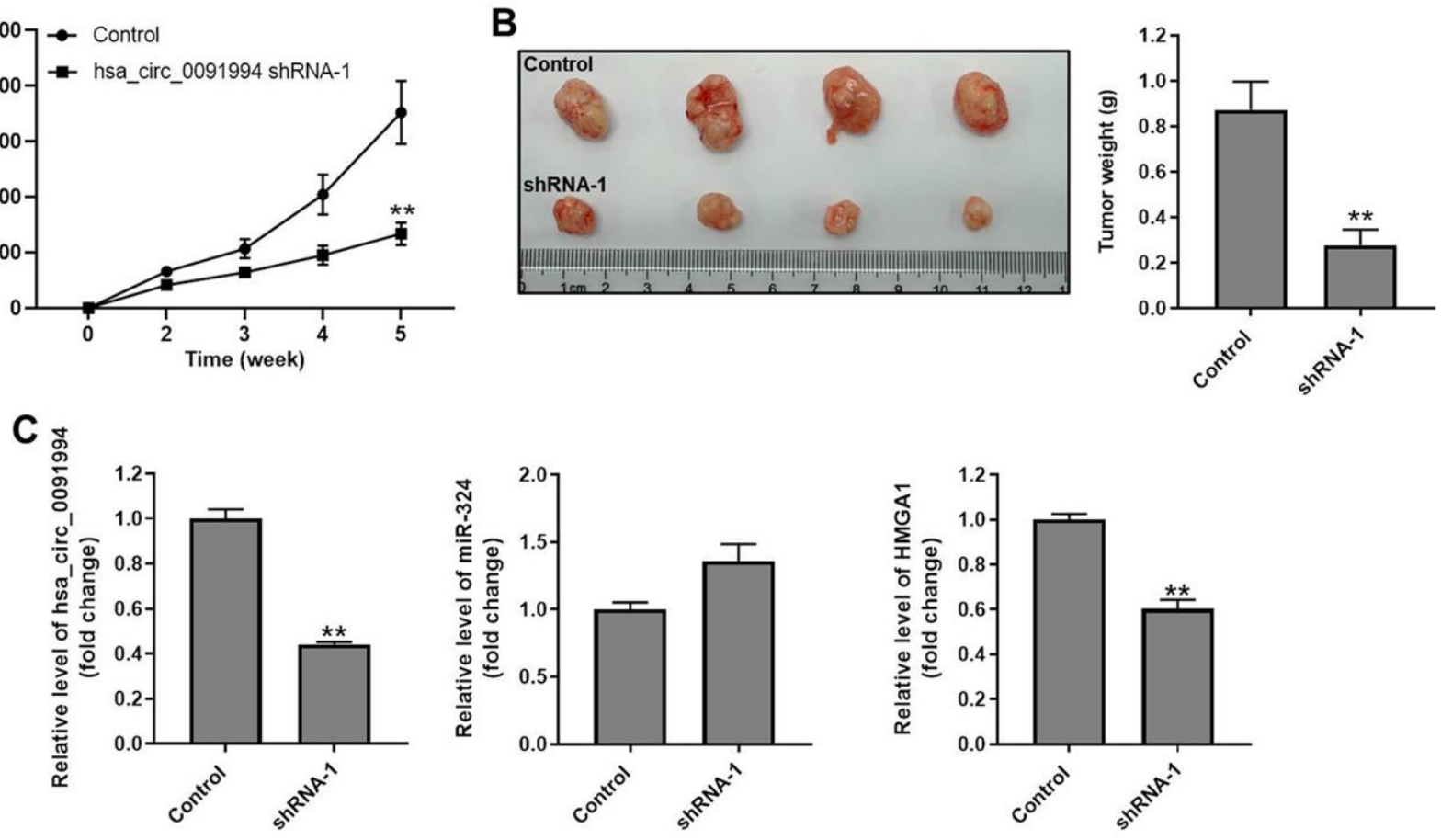

Figure 6. Hsa_circ_0091994 knockdown repressed GC tumor growth in mouse xenograft model. Hsa_circ_0091994 shRNA-1 treated AGS cells were injected into right dorsal of the mice ( $n=6$ per group). (A) Tumor volume was measured at weekly. (B) Tumor tissues were harvested and weighted at the end of week 5. (C) The expressions of hsa-circ-0091994, miR-324-5p and HMGA1 in tumor tissues were detected with RT-qPCR. ${ }^{* *} \mathrm{P}<0.01$, compared with control; $\mathrm{n}=6$. 
out with EnTurbo ${ }^{\mathrm{TM}}$ SYBR Green PCR SuperMix (ELK Biotechnology) on a StepOne ${ }^{\mathrm{TM}}$ Real-Time PCR System (Thermo Fisher Scientific). Thermal cycling protocol was pre-incubation at $95^{\circ} \mathrm{C}$ for $3 \mathrm{~min}$, followed by 40 cycles of $95^{\circ} \mathrm{C}$ for $10 \mathrm{~s}, 58^{\circ} \mathrm{C}$ for $30 \mathrm{~s}$, and $72^{\circ} \mathrm{C}$ for $30 \mathrm{~s}$. Relative expression was determined using the comparative $2^{-\Delta \Delta \mathrm{Ct}}$ method. U6 was used as the inner control for miRNA324-5p. $\beta$-actin acted as the inner control for other genes. Primers were listed as follows: $\beta$-actin, forward, 5'-GTCCACCGCAAATGCTTCTA-3', reverse, 5'-TGCTGTCACCTTCACCGTTC-3'; Hsa-circ0091994, forward, 5'-CGACCACCATGACAACACCT A-3', reverse, 5'-CCAGCAGCTTTGGCATTTAC-3'; Hsa-miR-324-5p, forward, 5'-CGCATCCCCTAGGGC AT-3', reverse, 5'-CTCAACTGGTGTCGTGGAGTC-3'; U6, forward, 5'-CTCGCTTCGGCAGCACAT-3', reverse, 5'-AACGCTTCACGAATTTGCGT-3'.

\section{Establish cell lines with stable hsa_circ_0091994 knockdown}

Short hairpin RNA (shRNA) against hsa_circ_0091994 and control shRNA (shRNA-ctrl) were synthesized and constructed into pcDNA3.1 vector by GenePharma (Shanghai, China). The pcDNA3.1 vector containing shRNA-ctrl, shRNA-1, or shRNA-2 was transfected into AGS and HGC-27 cells with Lipofectamine 2000 transfection reagent (Thermo Fisher Scientific). The transfection procedures were carried out per manufacturer's instructions. Stably infected cells were selected using $5 \mu \mathrm{g} / \mathrm{ml}$ neomycin (Sigma, Saint Louis, MO, USA). The sequences of shRNA were as follows: shRNA-1, 5'-TGTCCAGCAGGTGTCGATTCAAGA GATCGACACCTGCTGGACATTTTT-3'; shRNA-2, 5'-GGTGTCGAGCTTGGCAATTCAAGAGATTGCC AAGCTCGACACCTTTTT-3'.

\section{Overexpression of HMGA1 and hsa_circ_0091994}

The HMGA1 and hsa_circ_0091994 overexpression (HMGA1 OE and hsa_circ_0091994 OE) pcDNA3.1 vectors were constructed by Genepharma. AGS cells were transfected with pcDNA3.1-HMGA1-OE vector for $48 \mathrm{hr}$ using Lipofectamine 2000 transfection reagent (Thermo Fisher Scientific). MKN45 cells were transfected with hsa_circ_0091994 OE for $48 \mathrm{hr}$ as well.

\section{Cell viability assay}

Cell counting kit-8 (CCK-8) assay was carried out to evaluate cell viability. Cells from each group were treated, then the cells were placed into a 96-well plate at density of $5 \times 10^{3} /$ well. After $10 \mu \mathrm{l}$ CCK- 8 reagent (Beyotime) was added to each well, the cells were incubated with CCK-8 reagent at $37^{\circ} \mathrm{C}$ for $2 \mathrm{hr}$.
The absorbance at $450 \mathrm{~nm}$ was determined using a spectrophotometer.

\section{Evaluation of cell apoptosis}

The cells from each group were placed into 6-well plates at $3 \times 10^{5}$ cell/well and cultured overnight. Annexin V-FITC Apoptosis Detection Kit purchased from Sungene Biotech (Tianjin, China) was employed for annexin V-FITC/propidium iodide (PI) doublestaining, following the manufacturer's protocol. Apoptotic cells were detected on a BD AriallI flow cytometry system (BD Biosciences, Franklin Lake, NJ, USA).

\section{Scratch assay}

Wound scratch assay was employed to examine the wound healing activity of AGS cells and HGC-27 cells after indicated treatment. The cells were placed into 6well plate at $5 \times 10^{5}$ cells/well. Once the cells created a confluent monolayer, scratches were created using sterilized $10 \mu \mathrm{L}$ pipette tips. Then the cell monolayer was washed with PBS to remove cell debris. Wound gaps were photographed at $48 \mathrm{hr}$.

\section{Luciferase reporter assay}

The potential downstream miRNA target of hsa_circ_0091994 was predicted using Starbase (http://starbase.sysu.edu.cn/) and CircInteractome (https://circinteractome.nia.nih.gov/index.html) online databases. The potential downstream target of miRNA was predicted with TargetScan (http://www.tar getscan.org/vert_72/), miRWalk (http://zmf.umm.uniheidelberg.de/apps/zmf/mirwalk/micrornapredictedtarget. html), and miRDB (http://www.mirdb.org/). Luciferase reporter assay was performed to verify the potential targeting relationship mentioned above. All oligos were synthesized by GenePharm (Shanghai, China). The oligos containing wild type or mutant 3' UTR binding site of hsa_circ_0091994 were cloned into pMIR-reporter vector (Promega, Madison, WI, USA). Then, pMIR-reporter vector constructs, renilla luciferase reporter vector, and miR-324-5p agomir were co-transfected into AGS cells for $48 \mathrm{hr}$. Dual Luciferase Reporter Assay System (Promega, Madison) was used once the relationship between miR-324-5p and HMGA1 was verified. The results were presented as a ratio of firefly luciferase activity normalized to Renilla luciferase activity [23].

\section{RNA pull-down assay}

RNA pull-down experiment was conducted according to previous reference [23]. Briefly, M-280 Streptavidin magnetic beads (Thermo Fisher Scientific) were 
incubated with the biotinylated miRNA-324-5p probe and negative control probe for $2 \mathrm{~h}$ at room temperature. After that, gastric cancer cells were harvested and incubated with probe-coated beads at $4^{\circ} \mathrm{C}$ overnight. Finally, the RNA complexes were extracted with Trizol reagent (Thermo Fisher Scientific) and analyzed by PCR assay.

\section{Western blot}

The cells were lysed in RIPA buffer (Aspen Biotechnology, Wuhan, China) supplemented with Protease Inhibitor Cocktail (Roche, NJ, USA) for $5 \mathrm{~min}$. The concentration of total protein was determined using BCA protein assay kit (Aspen Biotechnology). Total protein samples $(40 \mu \mathrm{g})$ were separated by sodium dodecyl sulfate-polyacrylamide gel electrophoresis (SDSPAGE). Separated proteins were blotted onto polyvinylidene fluoride (PVDF) membrane (Millipore, Bedford, MA, USA). After blocking in 5\% skim milk at room temperature for $1 \mathrm{hr}$, the PVDF membrane was incubated with primary antibodies at $4^{\circ} \mathrm{C}$ overnight. The PVDF membrane was washed three times with trisbuffered saline and tween 20 (TBST) then incubated with HRP-Goat anti Rabbit secondary antibodies (1:10000, Aspen) for $2 \mathrm{hr}$ at room temperature. The blots were visualized with an enhanced chemiluminescence (ECL) kit (Aspen). $\beta$-actin was utilized a loading control. The primary antibodies for western blot were: HMGA1 (1:1000, Abcam, Cambridge, MA, USA), Bax (1:2000, Cell Signaling Technology, Danvers, MA, USA), Bcl-2 (1:1000, Abcam), cleaved caspase-3 (1:1000, Abcam), $\beta$ actin (Abcam, 1:1000).

\section{Tumor xenograft model}

Six week old BALB/c nude mice were obtained from Vital River (Beijing, China) and randomized into the control group and hsa_circ_0091994 shRNA-1 group ( $n=6$ per group). AGS cells were transfected with hsa_circ_0091994 shRNA-1 for $48 \mathrm{~h}$ and then subcutaneously injected into the right flanks of the mice. Tumor volume was measured using a caliper every week after inoculation (Figure 6A). At the end of week 5 , the mice were sacrificed, and tumor tissue was harvested (Figure 6B). The tumor weight was measured immediately after sacrifice (Figure 6B). The tumor volumes were calculated using $1 / 2$ (length $\times$ width $^{2}$ ). Meanwhile, the levels of hsa_circ_0091994, miR-3245p and HMGA1 in tumor tissues of mice were detected by RT-qPCR (Figure 6C).

\section{Statistical analysis}

The data were analyzed using GraphPad Prism 8.0 (GraphPad Software Inc., La Jolla, CA, USA). One-way analysis of variance (ANOVA) was utilized to examine the significance between groups. All data were presented as the mean $\pm \mathrm{SD} . P<0.05$ was a statistical difference.

\section{Ethics approval and consent to participate}

All animal procedures were approved by the ethics committee of Henan Provincial People's Hospital. The principles of the NIH Guide for the Care and Use of Laboratory Animals were strictly followed.

\section{AUTHOR CONTRIBUTIONS}

Yi Xie conceived and supervised the study; Yi Xie and Zhao Liu designed the experiments; Hanfang Zhu performed the experiments.

\section{CONFLICTS OF INTEREST}

The authors declare that they have no conflicts of interest.

\section{FUNDING}

This study was supported by Henan Provincial People's Hospital.

\section{REFERENCES}

1. Molina-Castro S, Pereira-Marques J, Figueiredo C, Machado JC, Varon C. Gastric cancer: Basic aspects. Helicobacter. 2017 (Suppl 1); 22:e12412. https://doi.org/10.1111/hel.12412 PMID:28891129

2. Röcken C. Molecular classification of gastric cancer. Expert Rev Mol Diagn. 2017; 17:293-301. https://doi.org/10.1080/14737159.2017.1286985 PMID:28118758

3. Charalampakis $\mathrm{N}$, Economopoulou $\mathrm{P}$, Kotsantis I, Tolia M, Schizas D, Liakakos T, Elimova E, Ajani JA, Psyrri A. Medical management of gastric cancer: a 2017 update. Cancer Med. 2018; 7:123-33. https://doi.org/10.1002/cam4.1274 PMID:29239137

4. Ferro A, Peleteiro B, Malvezzi M, Bosetti C, Bertuccio $P$, Levi F, Negri E, La Vecchia C, Lunet N. Worldwide trends in gastric cancer mortality (1980-2011), with predictions to 2015, and incidence by subtype. Eur J Cancer. 2014; 50:1330-44.

https://doi.org/10.1016/j.ejca.2014.01.029 PMID:24650579

5. Ansari S, Gantuya B, Tuan VP, Yamaoka Y. Diffuse Gastric Cancer: A Summary of Analogous Contributing Factors for Its Molecular Pathogenicity. Int J Mol Sci. 2018; 19:2424. 
https://doi.org/10.3390/ijms19082424

PMID:30115886

6. Youn GJ, Chung WC. [Micrometastasis in Gastric Cancer]. Korean J Gastroenterol. 2017; 69:270-77.

https://doi.org/10.4166/kjg.2017.69.5.270

PMID:28539031

7. Ferlay J, Soerjomataram I, Dikshit R, Eser S, Mathers C, Rebelo M, Parkin DM, Forman D, Bray F. Cancer incidence and mortality worldwide: sources, methods and major patterns in GLOBOCAN 2012. Int J Cancer. 2015; 136:E359-86.

https://doi.org/10.1002/ijc.29210 PMID:25220842

8. Lott PC, Carvajal-Carmona LG. Resolving gastric cancer aetiology: an update in genetic predisposition. Lancet Gastroenterol Hepatol. 2018; 3:874-83. https://doi.org/10.1016/S2468-1253(18)30237-1 PMID:30507471

9. Macdonald JS, Smalley SR, Benedetti J, Hundahl SA, Estes NC, Stemmermann GN, Haller DG, Ajani JA, Gunderson LL, Jessup JM, Martenson JA. Chemoradiotherapy after surgery compared with surgery alone for adenocarcinoma of the stomach or gastroesophageal junction. N Engl J Med. 2001; 345:725-30.

https://doi.org/10.1056/NEJMoa010187 PMID:11547741

10. Molaei F, Forghanifard MM, Fahim Y, Abbaszadegan MR. Molecular Signaling in Tumorigenesis of Gastric Cancer. Iran Biomed J. 2018; 22:217-30.

https://doi.org/10.22034/ibj.22.4.217

PMID:29706061

11. Berger H, Marques MS, Zietlow R, Meyer TF, Machado JC, Figueiredo C. Gastric cancer pathogenesis. Helicobacter. 2016 (Suppl 1); 21:34-38.

https://doi.org/10.1111/hel.12338

PMID:27531537

12. Li Z, Huang C, Bao C, Chen L, Lin M, Wang X, Zhong G, Yu B, Hu W, Dai L, Zhu P, Chang Z, Wu Q, et al. Exonintron circular RNAs regulate transcription in the nucleus. Nat Struct Mol Biol. 2015; 22:256-64.

https://doi.org/10.1038/nsmb.2959

PMID:25664725

13. Starke S, Jost I, Rossbach O, Schneider T, Schreiner S, Hung LH, Bindereif A. Exon circularization requires canonical splice signals. Cell Rep. 2015; 10:103-11.

https://doi.org/10.1016/i.celrep.2014.12.002

PMID:25543144

14. Du WW, Zhang C, Yang W, Yong T, Awan FM, Yang BB. Identifying and Characterizing circRNA-Protein Interaction. Theranostics. 2017; 7:4183-91.

https://doi.org/10.7150/thno.21299

PMID:29158818
15. Li R, Jiang J, Shi H, Qian H, Zhang X, Xu W. CircRNA: a rising star in gastric cancer. Cell Mol Life Sci. 2020; 77:1661-80.

https://doi.org/10.1007/s00018-019-03345-5 PMID:31659415

16. Fang X, Wen J, Sun M, Yuan Y, Xu Q. CircRNAs and its relationship with gastric cancer. J Cancer. 2019; 10:6105-13.

https://doi.org/10.7150/jca.32927

PMID:31762820

17. Shan C, Zhang Y, Hao X, Gao J, Chen X, Wang K. Biogenesis, functions and clinical significance of circRNAs in gastric cancer. Mol Cancer. 2019; 18:136. https://doi.org/10.1186/s12943-019-1069-0 PMID:31519189

18. Lin H, Zhou AJ, Zhang JY, Liu SF, Gu JX. MiR-324-5p reduces viability and induces apoptosis in gastric cancer cells through modulating TSPAN8. J Pharm Pharmacol. 2018; 70:1513-20.

https://doi.org/10.1111/jphp.12995

PMID:30159900

19. Gao J, Liu J. Study on the Effect and Mechanism of the Expression of High Mobility Protein $A 1$ and $A 2$ in Gastric Cancer on Invasion and Metastasis of Gastric Cancer. Chinese Medicine and Clinic. 2019; 19:889-92.

20. Tang Z, Li C, Kang B, Gao G, Li C, Zhang Z. GEPIA: a web server for cancer and normal gene expression profiling and interactive analyses. Nucleic Acids Res. 2017; 45:W98-102.

https://doi.org/10.1093/nar/gkx247

PMID:28407145

21. Liu H, Zhang $X$, Jin X, Yang $Y$, Liang G, Ma Y, Wang B. Long Noncoding RNA VPS9D1-AS1 Sequesters microRNA-525-5p to Promote the Oncogenicity of Colorectal Cancer Cells by Upregulating HMGA1. Cancer Manag Res. 2020; 12:9915-28. https://doi.org/10.2147/CMAR.S273687 PMID: $\underline{3116849}$

22. Jin GH, Shi Y, Tian Y, Cao TT, Mao Y, Tang TY. HMGA1 accelerates the malignant progression of gastric cancer through stimulating EMT. Eur Rev Med Pharmacol Sci. 2020; 24:3642-47.

https://doi.org/10.26355/eurrev 202004 20826 PMID:32329839

23. Wang $P$, Liu YH, Yao YL, Li Z, Li ZQ, Ma J, Xue YX. Long non-coding RNA CASC2 suppresses malignancy in human gliomas by miR-21. Cell Signal. 2015; 27:275-82.

https://doi.org/10.1016/i.cellsig.2014.11.011 PMID:25446261 


\section{SUPPLEMENTARY MATERIALS}

\section{Supplementary Figures}

A

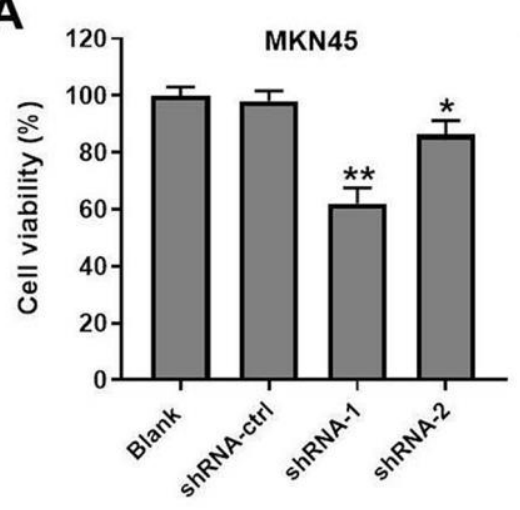

B

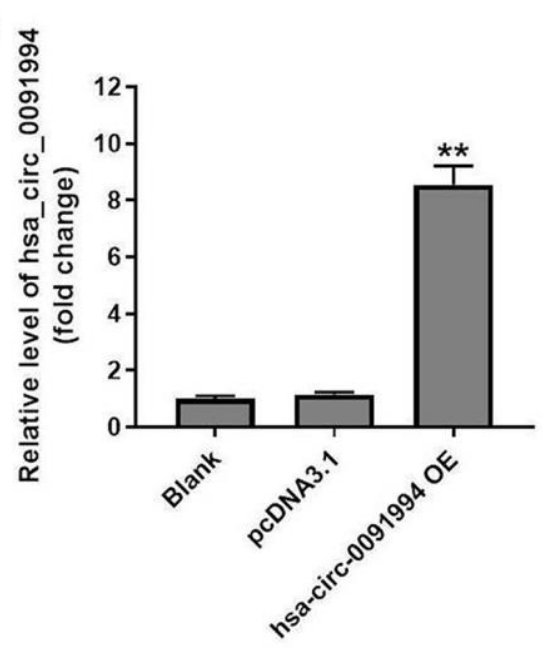

C

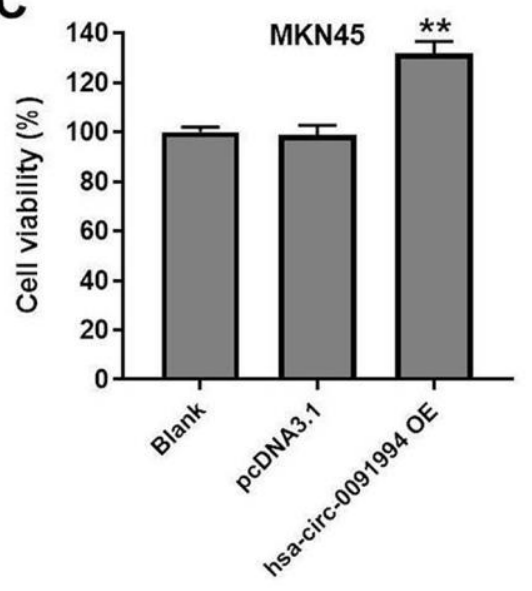

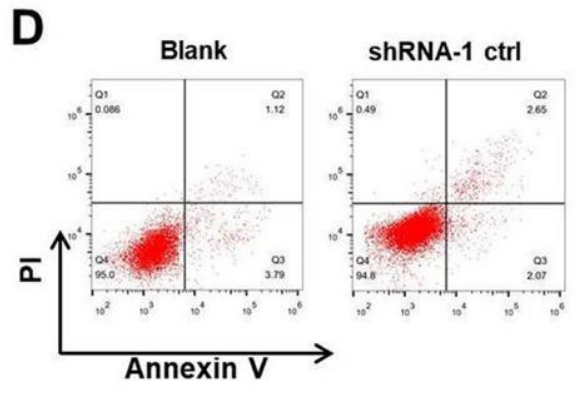
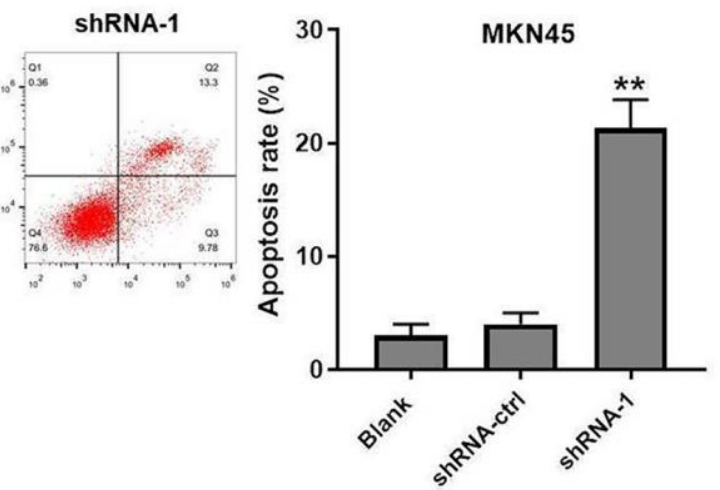

Supplementary Figure 1. Hsa_circ_0091994 knockdown inhibited the proliferation of MKN45 cells via inducing apoptosis. (A) MKN45 cells were administrated with shRNA-ctrl, shRNA-1 or shRNA-2 of hsa_circ_0091994 for 48 hr. The cell viability of MKN45 was determined by CCK-8 assay. MKN45 cells were administrated with hsa_circ_0091994 OE for 48 h. (B) The level of hsa_circ_0091994 was detected by RT-GPCR. (C) The cell viability of MKN45 was determined by CCK-8 assay as well. (D) MKN45 cells were administrated with shRNA-ctrl, shRNA-1 of hsa_circ_0091994 for $48 \mathrm{hr}$ and cell apoptosis was measured in each group. ${ }^{*} \mathrm{P}<0.05,{ }^{*} \mathrm{P}<0.01$, compared with blank; $\mathrm{n}=3$. 

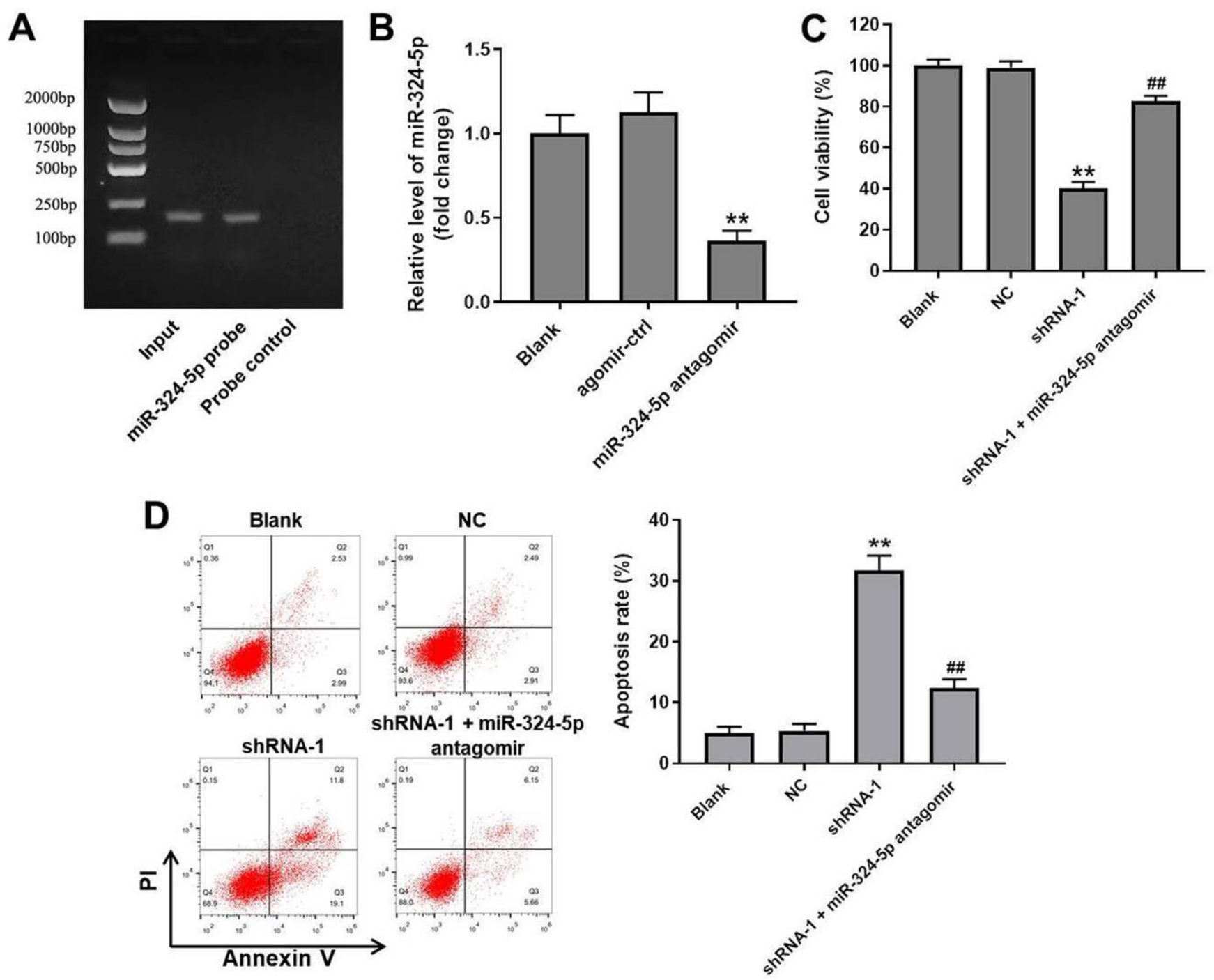

Supplementary Figure 2. The effects of hsa_circ_0091994 knockdown on the proliferation and apoptosis of MKN45 cells were reversed by miR-324-5p antagomir. (A) The interaction between hsa_circ_0091994 with miR-324-5p was detected with RNA pull down assay. (B) MKN45 cells were treated with miR-324-5p antagomir for $48 \mathrm{~h}$; the level of miR-324-5p was measured with RT-qPCR. MKN45 cells were treated with hsa_circ_0091994 shRNA1 or/and miR-324-5p antagomir for 48 h. (C) Cell viability was measured with CCK8 assay. (D) Cell apoptosis was measured in each group. ${ }^{* *} \mathrm{P}<0.01$, compared with blank; $\mathrm{n}=3$. 